\title{
Varietäten als Gegenstand der Verhandlung in Texten sogenannter Südschleswiger Literatur
}

\begin{abstract}
An wen richtet sich eigentlich die ,Südschleswiger Literatur“, von der meist als Regionalliteratur angenommen wird, dass sie in einem defizitären, unter deutschem Einfluss stehenden Dänisch verfasst sei? Der Umstand, dass die Funktionäre, Minderheitsangehörigen und die Sympathisanten (als potentielle Neuzugänge der Minorität) ganz unterschiedliche Gewohnheiten und Fertigkeiten des mündlichen und schriftlichen Sprachgebrauchs haben, findet nur allzu zögerlich Anerkennung. Die Diglossien der genealogisch motivierten und der neu zugeschriebenen Minoritätsmitglieder weichen voneinander ab und setzen unterschiedliche Koordinaten von Standard- und Nicht-Standard-Varietäten fest, die aber keineswegs mit der geographischen Herkunft korrespondieren müssen. Dieser transnational-translokale Bezug ist als deterritoriales Merkmal ebenso interessant wie der historisch früh verwendete Begriff der „Gesinnungsfreiheit“, der Geburts- bzw. Wohnort, Nationalität und Sprachpräferenzen voneinander entkoppelt und auf diese Weise eine interessante Vorstufe zum heutigen Prinzip der Selbstzuschreibung darstellt. Das analysierte dänische Gedicht (1977) handelt von Spracherwerb und nationaler Identifikation, wobei das lyrische Ich den Zwang zur Anwendung einer ,homogenen dänischen Standardvarietät‘ internalisiert hat. Das Thema des Hin- und Her-Übersetzens, das positionierte Sprechen in hegemonial geprägten Situationen sowie vielfältige Varietäten-Konstellationen zeichnen indes die untersuchte Revue (2016) aus. Diese adressiert Vertreter der Minorität, der dänisch- und der deutschsprachigen Majorität auf jeweils unterschiedliche Weise, was eine Reihe von metapragmatischen Kommentierungen ermöglicht.
\end{abstract}

Keywords: Adressierung; Positionierung; Dänisch; Lernersprachen; Mehrsprachigkeit; Sprachvarietäten; Südschleswig; Südschleswiger Literatur; Sønderjysk

Die sogenannte Südschleswiger Literatur weist einen charakteristischen doppelten Adressatenbezug auf: Es wird zunächst die vorgestellte Gemeinschaft der Lesenden angesprochen, die sich selbst der dänischen Minderheit zurechnet. Weiters richtet sich diese thematisch motivierte Gattung an Leser dänischer Literatur allgemein. Wie stark diese allgemeine Gruppe von nationalliterarischen Koordinaten oder von einem Bewusstsein für Varietäten bzw. für das hegemoniale Gefälle zwischen dem ,kolonialen Mutterland‘ Dänemark und der däni- 
schen Minorität geprägt ist, kann nur anhand konkreter Fallbeispiele ermessen werden. ${ }^{1}$

Der erste Adressatenbezug betrifft vor allem die Funktion der Sprachpflege sowie eine identitätspolitische Agenda. Mit der Begriffsprägung „Südschleswiger Erinnerungsliteratur“ wird eine historische Vergewisserung der „wir“-Gemeinschaft angestrebt. Die zweite elementare Funktion der ,Südschleswiger Literatur ist stärker auf die Zukunft bezogen, auf den Appell an die Förderinstitutionen Dänemarks und Deutschlands: Eine aktive Literatur- und Kulturszene repräsentiert den erfolgreichen Fortbestand dänischer Nationalkultur in der Grenzregion und lebt vor, wie erhaltenswert oder gar ausbaufähig sowohl die Literatur selbst als auch ihre Vermittlungsinstanzen sind.

Die Aufschlüsselung der Konstellationen von Sprachen/Varietäten als temporären relationalen Gefügen in einem vorgestellten Sozialraum scheint reizvoller als eine Herleitung von ,Sprachbesitztümern“, die sich aus den Grenzverschiebungen der komplizierten dänisch-deutschen und schleswig-holsteinischen Geschichte ergeben. Um die Relationen und sozialen Positionierungen jenseits territorialisierter Sprachkategorien erfassen zu können, ist das gleichzeitige Zusammenwirken der Varietäten mit indexikalischen Registern zu betrachten: Register sind internalisierte Modelle, in denen spezifische Sprechweisen indexikalisch auf bestimmte Eigenschaften von Personen verweisen (vgl. Agha 2007, 134-136). Die Registrierung bezeichnet die Verfestigung eines Registers (vgl. Agha 2007, 81), das über Stereotypisierungen wiedererkannt und abgerufen wird. Mit der Aufschlüsselung jener sprachideologischen Prozesse kann vermieden werden, essentialistische Konzepte nahezulegen oder die Festschreibung bestimmter Varietäten in einer Unterlegenheits- bzw. Dominanzposition unreflektiert zu bestätigen.

Im Folgenden sollen zwei Textbeispiele auf ihre Adressatenbezüge, Varietäten-Konstellationen (vgl. Fredsted 2016, 438) und sprachideologische Markierungen hin befragt werden: eine Revue aus dem Jahr 2016, die derzeit noch auf dem Spielplan steht, und ein wiederentdecktes Gedicht von 1977.

1 Für die redaktionelle Überarbeitung sämtlicher Beiträge der ICLA-Sektion „Stylistic Phenomena in Multilingual Literature since 1900“ (22.-23.7.2016 unter der Leitung von Antje Wischmann, Johanna Laakso, Hannah Tischmann und Philipp Wagner) danke ich der Praktikantin der Abt. Skandinavistik am Institut für Vergleichende Europäische Sprach- und Literaturwissenschaft der Universität Wien, Amrei Stanzel, sehr herzlich. 


\section{Durchgespielte Szenarien}

Die Revue von Kaj Nissen: Søsteren der ikke vendte hjem. Et stykke om Sydslesvig [Die Schwester, die nicht heimkehrte. Ein Stück über Südschleswig] wurde im dänischen Theater Flensborg, „Det lille Teater“, im Januar 2016 uraufgeführt und steht immer noch auf dem Spielplan (vgl. Nissen 2016). Der Verfasser Nissen (geboren 1941 in Sønderborg) hat sich durch historische Literatur in unterschiedlichen Genres wie Drama, Hörspiel und Kinder- und Jugendbuchliteratur einen Namen gemacht (vgl. Davidsen 2014). Er ist in den „reichsdänischen“ Kanon aufgenommen und nennt sich selbst zweisprachig, bezogen auf die beiden nationalsprachlichen Standardvarietäten. Dennoch bietet die Revue mit Musikund Gesangseinlagen über die „kleine Schwester Südschleswig“ als einem personifizierten Landesteil, dem eine „Heimkehr“ ins Mutterland versagt blieb, ein beachtliches Aufgebot an Varietäten. Das „aufeinander ausgerichtete“ Sprechen (Spitzmüller 2014) tritt in der Revue auf mehreren Ebenen hervor. Bereits das Lesedrama bietet eine Reihe von metapragmatischen Kommentierungen, die sich bei jeder neuen Aufführung performativ auffächern.

Die Revue brilliert mit einem postkolonialen Auftakt: Eine historische Wanderlehrerin führt als Moderatorin durch einen bunten Szenenreigen, der grob an ausgewählten Stationen der Schleswiger Geschichte orientiert ist. Die ambulierenden Lehrerinnen haben in der Geschichte der Minderheit Heldinnenstatus, denn sie treten sowohl befreiend (im Kontrast zum preußischen System) als auch missionierend für eine nationale Erziehung zum ,Dänentum‘ auf. Die historische politische und sprachliche Danisierung, die im neunzehnten und zwanzigsten Jahrhundert verwirklicht werden sollte, erscheint heute angesichts des jüngst reformulierten Einsprachigkeitszwangs in einem neuen Licht: Die Lehrerinnenfigur betritt nämlich den Theatersaal, als ob sie in ein Klassenzimmer käme. Schlimmer noch: Sie kündigt dem Publikum Dänisch-Anfängerunterricht an. Die Bestrebung, eine nationale Haltung zu reformulieren und diese mit der zu verwendenden (National-)Sprache zur Deckung zu bringen, wird ironisch bis skeptisch distanziert betrachtet.

Die theatrale Räumlichkeit der Bühne selbst wird nicht für eine territoriale Semantisierung genutzt, die auf Topographie oder Karten verweisen könnte. Hierdurch verstärkt sich die Aufmerksamkeit auf das Zusammenspiel der Varietäten. Das Stück legt Zeugnis davon ab, dass multikulturalistische Topoi auf Sprachenverhältnisse umgelegt worden sind. Aus der Perspektive eines verallgemeinerten „wir“ formulieren nummerierte Vertreter metapragmatische Kommentare. Die Szene ist als Chor der Vielfalt arrangiert: 
[...] ACHT: Hier nimmt keiner die Unterschiede zwischen den Sprachen besonders ernst. EINS: Man spricht Deutsch mit dänischen Wendungen oder Dänisch mit deutschen Wendungen.

ZWEI: Man wechselt die Sprachen, wie andere ihre Hemden wechseln - manchmal, ohne es zu merken.

DREI (skeptisch): Und beim Wechseln der Sprache werden dann ganz von allein die kulturellen Codes umgestellt?

VIER: Es ist nicht verwirrend, sich in die Sprachen hinein- und aus ihnen herauszubewegen. [...] EINS: Dänisch hat in Südschleswig den Status einer offiziellen Sprache erhalten! Jeder hat das Recht, sich in Wort und Schrift in dänischer Sprache an die Behörden zu wenden! ${ }^{2}$

Mit der Frage des Sprechers Drei nach den kulturellen Codes im Verhältnis zu sprachlichen Codes wird produktiv ein sprachideologischer Entwurf von codeswitching in Zweifel gezogen, der Sprache und Kultur in einem unmittelbaren Bedingungszusammenhang konzeptualisiert (vgl. Fredsted 2016, 439). Indes wird die im Laiendiskurs gängige Annahme bestätigt, dass Nationalsprachen geschlossenen Systemen glichen (hinein - hinaus). Die letzte Feststellung von Figur Eins nennt wiederum eine symbolische Verwendung von Mehrsprachigkeit, nämlich die Policy Schleswig-Holsteins für den Umgang mit Minderheitensprachen.

Schon mit dem Revuetitel und durch die Diskursinstitution „Det lille Teater“ wird eine „homogenizing convention“ (Sternberg 1981) nahegelegt. Die Literatursprache Dänisch stellt die maßgeblichen Wissensrahmungen bereit und gibt einen Standort übergeordneten Sprechens vor. Besuchen etwa Touristen aus Jütland das Theater, würde in erster Linie die metapragmatische Kommentierung ,nicht-standarddänischer` Varietäten legitimiert.

Die Lernersprache der Neuzugänge zur dänischen Minderheit wird innerhalb der Minderheiteninstitutionen kontrovers verhandelt; in den Mitgliederzeitschriften und Aussendungen lässt sich ein breites Spektrum an offiziellen Verlautbarungen, aber auch an verdeckten oder halböffentlichen Stellungnahmen ausmachen. Die Zuschauer werden - womöglich exkludierend - in sozialen und sprachideologischen Distinktionen geschult, wenn sie etwa herauszufinden suchen, ob es sich bei den Schauspielern/Rollenfiguren um geübte oder ungeübte Varietätenanwender handelt.

2, „[...] OTTE: Ingen her tager skellene mellem sprogene særligt højtideligt.

ET: Der tales tysk med danske vendinger eller dansk med tyske vendinger.

TO: Man skifter sprog som andre skifter skjorte - nogle gange uden at lægge mærke til det.

TRE (skeptisk): Og med sproget følger alle de kulturelle koder af sig selv?

FIRE: At bevæge sig ind og ud af sprogene er ikke noget at lade sig forvirre af.

[...] ET: Dansk har fået status som officiellt sprog i Sydslesvig! Enhver har fået ret til at henvende sig til myndighederne på dansk i skrift og tale!“ (Nissen 2016, 67). 
Die sogenannte Authentizität des Sprachgebrauchs kann durch spezifische Interferenzphänomene bei einzelnen Schauspielern unterlaufen werden, da diese sich unter Umständen nur temporär einer (ungewohnten) Varietät bedienen. Dadurch wird eine besondere Spielart der Multiglossie angebahnt. Auch wenn das bisherige Wissen der Zuschauer und Mitwirkenden um Register keineswegs getilgt wird, können im Zusammenwirken der Varietäten neuartige relationale Bedeutungen entstehen.

Im Hinblick auf die soziolinguistische Sensibilisierung besticht die Marionettenszene aufgrund ihres Komplexitätsgrads. In der Gegenüberstellung zweier Marionetten wird die Ebenbürtigkeit von Sønderjysk als einem dänischen Dialekt und der Südschleswiger-Varietät (im Folgenden SSL-Varietät) als einem „mit dem Deutschen vermischten dänischen Dialekt“ demonstriert (linguistisch nicht haltbar). Sønderjysk und die SSL-Varietät seien Pendants. Hiermit scheint der metalinguistische Kommentar zu bestätigen, dass diese „Dialekte“ auf klar abgrenzbare geographische Gebiete verwiesen - und nicht etwa auf sprach- oder sozialräumliche Domänen.

Die Marionetten geraten in einen Streit über die Varietäten, die sie selbst verkörpern. Die Figuren werden auf der Bühne von der großen Schwester Nordschleswig und der kleinen Schwester Südschleswig gelenkt; die Puppen sind laut Regieanweisung in Trachten der beiden Regionen gekleidet. Puppe 2 spricht Sønderjysk oder Jütisch, offenbar abhängig von den Fertigkeiten der Schauspieler, Puppe 1 die SSL-Varietät (vgl. Nissen 2016, 49). Der Streit stellt die territoriale Konzeptualisierung und die Statusindikatoren zur Debatte:

PUPPE 1: [...] und hieß nicht eigentlich das gesamte Gebiet Schleswig, als es noch ein Herzogtum war?

PUPPE 2: Wie kann sie doch reden! Jetzt ist sie ja richtig in Fahrt geraten!

PUPPE 1: Was sagst du? Red' mal Dänisch mit mir!

PUPPE 2: Mach' ich doch! Wir haben schon immer Sønderjysk gesprochen, das ist nun wirklich ,Urdänisch'!

PUPPE 1: Ja, so ein Halbdeutsch - oder eher eine Art Plattdeutsch für Anfänger!

PUPPE 2: Das musst du gerade sagen, du mit der südschleswiger Sprache!

[...]

WANDERLEHRERIN: Kein Sønderjysk mehr bitte, übersetze es bitte ins Reichsdänische!

PUPPE 2: Sprich doch mal altmodisches Dänisch - falls du das überhaupt kannst! ${ }^{3}$

3 „DUKKE 1: [...] og hed det for resten ikke også Slesvig da det hele var hertugdømme?

DUKKE 2: Vår ka' ho’ snak! Dæ’r da retti kåmmet knal’å!

DUKKE 1: Hvad sir' du? Tal dog dansk til mig!

DUKKE 2: Det gør [jeg, sic] osse! Vi har alltid snakket synnejysk, det er ærkedansk, er det!

DUKKE 1: Sådan lidt halvtysk - eller er det plattysk for begyndere! 
Puppe 1 setzt sich zur Wehr und stellt Sønderjysk als defizitäres Pidgin dar, indem erstens eine abwertende Analogie zwischen Plattdeutsch und Sønderjysk hergestellt wird, als wären es „,ausgestorbene“ volkstümliche Mundarten. Zweitens wird die „Halbsprache“ eingeführt, welche den Topos einer „monolingualen Ganzheit“ aufgreift, drittens die Lernersprache von Anfängern. Indem die Puppen an Fäden hängen, erhalten die Dialekte keinen autonomen Akteurstatus, ihre sprachideologische Positionierung bleibt von nationalsprachlichen Koordinaten bestimmt.

Die Wanderlehrerin wird dadurch als „Reichsdänin“ charakterisiert, dass sie Sønderjysk gar nicht versteht; dies ebenfalls eine metalinguistische Setzung, die zugleich den mentalen Abstand der Missionare zu den Kolonialisierten betont. Die gesamte Szene wird von ihr als ein Sprachkurs bezeichnet und so mit einer sprachideologischen Wissensrahmung versehen. Bei ihrer Geschichtsvergewisserung legt sie Wert auf ein repräsentatives Verhalten der Südschleswiger Tochter Dänemarks, indem sie betont, dass jene unter Beobachtung stünde: „Denk dran, die Leute sehen und hören zu!“ („Husk nu at folk ser på dig, folk lytter!“ Nissen 2016, 60). Dieser selbstbezügliche Scherz über das Spiel im Spiel gemahnt zugleich an die „Repräsentationspflicht“ des dänischen Theaters, wenn auch satirisch abgefedert.

Die Rivalität der Sprachvarietäten als dialektalen Machtkampf von Marionetten darzubieten, stellt in der Terminologie Sternbergs eine „conceptual reflection“ und „explicit attribution“ dar (Sternberg 1981, 230-232). Beide Verfahren setzen eigentlich eine hohe Sprachkompetenz der Rezipienten voraus (vgl. Sternberg 1981, 233). Indem die Lehrerin die überwachende „Beobachtung“ insbesondere derjenigen Puppe erwähnt, die die SSL-Varietät verwendet, wird ein Tabubereich gestreift, nämlich die interne Sprachzensur.

\section{Sinnstiftende Differenz}

Die literarische Produktion Karin Johannsen-Bojsens (geboren 1936) steht im Zeichen des politischen Engagements der dänischen Minderheit seit der Nachkriegszeit. Ihre Werke stellen ein Gründungsnarrativ für diejenigen Minderheitsangehörigen bereit, die sich zum Zeitpunkt ihrer identitätspolitischen Zuschrei-

DUKKE 2: Du sku’ nødig snakke sku’ du! Du med dit sydslesvigske sproch! [...] VANDRELÆRERINDE: Ik' mer’ synnejysk, tak - prøv at oversæt det til rigsdansk! DUKKE 2: Tal nu gammeldags [kursiv im Original] dansk - hvis du ellers er i stand til det!“ (Nissen 2016, 50-51) 
bung als vornehmlich deutschsprachige Personen auffassten. Die bis 1945 übliche Rückverlängerung der Minderheitsgeschichte auf das seit 1864 bestehende nationale Spannungsfeld im Grenzland wird dadurch relativiert, wenn nicht gar unterbrochen, und ein sogenanntes binationales Denkmodell eingeführt. Dieses weist mitunter der Figur des „Dritten“ eine starke Position zu - dem sogenannten Südschleswigertum. Aus heutiger Sicht fallen die multikulturalistischen Grundannahmen ins Auge, die sich allerdings in der Auseinandersetzung mit Mehrsprachigkeitskonstellationen tendenziell auf ein Konzept der Diversität hinzu bewegen.

Mit dem Akt der sprachlichen und identitätspolitischen Zuschreibung setzt sich Sindelag [Gesinnung (1977)] auseinander, eine Gedichtsammlung mit autobiographischen Reminiszenzen, die zum 75. Geburtstag der Autorin 2011 neu aufgelegt und als Südschleswiger Erinnerungsliteratur kanonisiert wurde. Gerade dieser Kanonisierungsvorgang ist aufschlussreich, war es doch Johannsen-Bojsen selbst, die seit den 1980er und 1990er Jahren als eine besondere Fürsprecherin der Gattung „Südschleswiger Literatur“ auftrat. Zu diesem Zeitpunkt hatte das literarische Feld dieser als Lokal- und Herkunftsliteratur definierten Gattung seine größtmögliche Autonomie erreicht, und die identitätspolitischen Minderheitendiskurse trugen zur Verbreitung und Popularität der ,eigenen` Südschleswiger Literatur bei.

Gerade vor dem identitätspolitischen Fond betont das ausgewählte Gedicht „Sproget“ (Johannsen 1977, 80-81, und Andersen 2015, 80-81) die Komplexität des Sprachgebrauchs, wobei die Temporalität, verschiedene Zweckorientierungen und eine Unterscheidung von institutioneller, gruppenspezifischer und privater Sprachverwendung hervorzuheben sind. Bezeichnenderweise bleibt - ganz anders als in Nissens Revue - die grundlegende Differenzachse Dänisch versus Deutsch in diesem Text unerwähnt, wenn sie nicht sogar temporär stillgelegt erscheint.

Das Gedicht nimmt den Sommeraufenthalt jugendlicher Minderheitsangehöriger in Dänemark zum Ausgangspunkt, der für die Verwender der SSL-Varietät eine zeitlich und lokal bestimmte Einweisung in die Konstituierung von Identität und Alterität bedeutet. Die metapragmatische Kommentierung bestimmt die Konstellation beider dänischer Varietäten mittels einer Sonderform des ,vehicular matching“ (Sternberg 1981, 223). Eingebettet in einen dänischen Textabschnitt werden in Strophe 5 drei dänische Substantive in Anführungszeichen wiedergegeben. Schriftsprachlich wird keine Unterscheidbarkeit impliziert, abgesehen von der umgangssprachlich dargebotenen Kurzform „Berlinger“ für die dänische Zeitung Berlingske Tidende. Erst wer diese verschliffene Kurzform kennt, ist in der Lage, diesen Namen als Zeitungstitel zu identifizieren. Durch den Aufenthalt werden die Jugendlichen an den mündlichen Gebrauch der Standardvarietät und an neue sprachlich-kulturelle Wissenseinheiten herangeführt; Berlingske Tidende ist eine landesweit erscheinende Zeitung. 
Die Einweisung in den Sprachgebrauch hat mithin nicht nur alltagspraktische Konsequenzen, sondern ist mit dem Aspekt sozialer Mobilität konnotiert und geht mit der Vorstellung translokaler Verknüpfungen einher. Durch die Kontextualisierungsoptionen ergeben sich (mindestens) zwei Interpretationen respektive Übersetzungen:

\author{
Wörter wie ,Berlinger` [landesweite dän. Tageszeitung], ,brød‘ [Brot], ,cykel` [Fahrrad] \\ waren für euch ohne Magie, \\ aber Zauberformeln für uns: \\ wo man sie verwendete, lebte man dänisch.
}

\title{
Alternativübersetzung:
}

Wörter wie ,Berlinger', ,Brot‘, ,Fahrrad“

waren für euch ohne Magie,

aber Zauberformeln für uns:

wo man sie verwendete, lebte man dänisch (Johannsen 1977, 81). ${ }^{4}$

Die erste Version bleibt insofern problematisch, als sie eine Varietätendifferenz in eine ,nationalsprachliche' Differenz transformiert und vermutlich damit eine Vergrößerung des Maßstabs vornimmt. Die zweite Version vollzieht nach, dass es keine nationalsprachliche Schwelle gibt und sich die Differenz erst Eingeweihten erschließt, die mit der Registrierung bereits Erfahrungen gesammelt haben.

Obwohl sich die ,Südschleswiger“ und die „reichsdänische“ Sprechergruppe offiziell der gleichen Nationalsprache bedienten, bietet zuallererst ihr unterschiedlicher Sprachgebrauch ein Distinktionsmerkmal. Die SSL-Varietät zirkuliert vor allem zwischen den Diskursinstitutionen, zu denen die - ebenfalls in der umgangssprachlichen Kurzform genannte - „Avis“ („Flensborg Avis“)

\footnotetext{
4 ,(1) Helt/ kunne vi aldrig nå hinanden// (2) For skønt vi år for år/ vandt mere hemmeligt terræn/ (som var det ingen sag)/ i jeres danske verden/ og brugte jeres danske sprog,/ som om vi havde mesterbrev/ (og ikke lærlingestatus),/ var det dog netop sproget,/ som skilte os fra jer.// (3) Hver gang, vi kom til sommerlandet,/ betjente I jer/ (med den retmæssige ejers/ naturlige sikkerhed)/ af nye talemåder,/ som holdt os udenfor.// (4) Vore egne gloser/ var som slidte mønter,/ stadig ud- og indbetalt/ hos de lokale kultursparekasser/ (skoler, foreninger, bibliotek og ,Avis')/ og vekslet af de samme folk,/ mens de kom ud af kurs i landet,/ de var præget i.// Værre end denne bestandige halten bagefter/ (og sværere at forklare)/ var, at selv om vi med tiden/ erobrede de nye ord,/ blev vi dog ved med at føle/ forskelligt fra jer.// (5) Ord som ,Berlinger‘, ,morgenbrød‘, ,cykel'/ var uden magi for jer,/ men trylleformler for os:/ hvor de brugtes, levede man dansk.// (6) Luften omkring dem blev mild.// Da toget igen/ kørte os ud af sommerlandet,/ lod vi, som var vi herre over disse ord,/ og viste dem pralende frem for hinanden/ som billet til danskernes verden.// (7) Men os selv kunne vi ikke snyde./ Vi vidste, vort vinterår ville være/ uden disse ord/ og det, de stod for.“(Johannsen 1977, 80-81).
} 
gehört, was schlicht „Zeitung“ bedeutet. Diesen Zeitungstitel können nur die mit den Flensburger Lokalitäten und Kultureinrichtungen Vertrauten erkennen, nicht etwa deutschsprachige Leser allgemein. Die umgangssprachlichen Benennungen der beiden Zeitungen verweisen auf die vorgestellten Gemeinschaften von Lesern, was für die literarische Vision einer „Südschleswiger“ Literatur mit doppeltem Adressatenbezug aussagekräftig ist. Es werden zwei sich überschneidende Lesergemeinschaften konstituiert, die sich für sowohl journalistische als auch literarische Texte in beiden genannten Varietäten interessieren. Dabei ist nicht unerheblich, dass es sich um die anerkannte einsprachige dänische Zeitung mit Verlagssitz in Kopenhagen handelt, im Unterschied zur provinziellen Flensborg Avis, die als eine der Minderheiteninstitutionen eingeführt ist, die im Gedicht als Kultursparkassen bezeichnet werden (vgl. Strophe 3). Mit dem Distributionsradius dieser Blätter wird ein Kontrast zwischen der ereignis- und textreichen Hauptstadt und dem stillen Provinzort heraufbeschworen, der sich auf das Verhältnis der dänischen Nationalliteratur zur „Südschleswiger“ Regionalliteratur übertragen lässt. Auch letztere kann entweder „,angeschlossen“ oder exkludiert werden.

Der regionalen Lesergruppe wird symbolische Priorität eingeräumt, indem die Minderheitenzeitung zuerst genannt ist. In der Kontrastierung der sozialen Stile ist die Berlingske Tidende, die sich in den 1970ern vor allem an das hauptstädtische konservative Bildungsbürgertum richtete, der Flensburger Zeitung, deren Leser damals mit einem sozialdemokratisch-kleinbürgerlichen Habitus konnotiert wurden, an Prestige weit überlegen.

Mit dem schwierig zu übersetzenden „morgenbrød“ [Brot zum Frühstück] wird die Gruppe der Kleinfamilie aufgerufen, vielleicht sogar eine für Dänemarktouristen vertraute sommerliche Situation. Wie werden touristischer Enthusiasmus und identifikatorische Nostalgiestimmung aus der Perspektive des „wir“ noch übertroffen? Der Brotverzehr am Morgen ist im Gedichtband mit Konnotationen eines existenziellen Neubeginns in der Nachkriegszeit versehen, eng verbunden mit dem Konzept überlebensnotwendiger ,Sprachnahrung“.

Was die Wissensdistribution an die ,reichsdänischen“ Adressaten betrifft, so lernen diese in Strophe 1 bis 4 einige Rahmenbedingungen der Minderheit kennen, bevor das Gedicht ein Sprachmerkmal aufgreift, das gemeinhin mit einer Überlegenheitsposition verknüpft ist: ein umfassendes sprachliches Repertoire für ein breites emotionales Spektrum. Dabei ist es die SSL-Varietät, die zur Erfahrung der Sprachmagie, zum Hunger auf Sprache und zu einer fundierten Sprachreflexion ermächtigt! Sie ermöglicht den Sprechern Differenzerfahrungen, die monolingualen Anwendern verborgen bleiben. Die geläufige nationalsprachliche Differenz wird geleugnet, so als gäbe es zwischen Dänisch und Deutsch keine wesentlichen sprachlichen oder sozialen Distinktionen. Indem dieser potentielle Antagonismus 
ausgehebelt erscheint, wird den beiden Nationalsprachen auch der ideologische Einfluss auf die Relation der Standard- und Nicht-Standardvarietät abgesprochen. Jegliches Revanchismus-Risiko wird hiermit wie beiläufig gebannt; der Spielraum der sogenannten Bonn-Kopenhagener Erklärungen („Erklärung der Regierung der Bundesrepublik Deutschland“ 1955) scheint lebendig veranschaulicht. In die Decodierung von Ausrichtung, Bewertung und Positionierung haben die SSLSprechenden mithin den größten Einblick.

Das naturalisierte Deutungsvorrecht der „reichsdänischen“ Sprecher wird in Frage gestellt, und der Muttersprachenmythos findet gar nicht erst Erwähnung. Die sprachlichen Elemente werden in Dänemark zwar wie Münzen geprägt, aber sie haben die Funktion eines Zahlungsmittels, eines Mediums und Speichers.

Mit den Metaphern der Kultursparkassen und der restringierten Valuta sind sämtliche Kapitalarten im Sinne Bourdieus vertreten: das kulturelle Kapital, das durch Schule und Bibliothek zugänglich wird, das soziale und schließlich auch das symbolische Kapital, das sich mit „Avis“ und dem dänischen Vereinsleben in Südschleswig erschließt. Bezeichnenderweise ist die Differenz zwischen ökonomischem und kulturellem Kapital im Neologismus „Kultursparkasse“ aufgehoben, wodurch die Interdependenz von kulturellem und ökonomischem Diskurs eingängig herausgestellt wird. Johannsen-Bojsen verfasst seit 1977 selbst „Funktionärsprosa“, die die Zirkulation zwischen den lokalen Abnehmern und den Adressaten einer umfassenden gesamtdänischen Leserschaft anreichert. Engagiert bemüht sie sich, eine Minoritätsliteratur im Auftrag der Gedächtnispflege fortzuschreiben (vgl. Johannsen-Bojsen 2016). Diese planvolle Arbeit am Kanon zielt aber immer auch auf eine Bestandssicherung der Minderheitsinstitutionen. Die „ideology broker“ (Jan Blommaert) können das umfangreiche Werk Johannsen-Bojsens als Beleg nicht nur für den literarischen Ertrag und die erfolgreich geleistete Sprachpflege instrumentalisieren, sondern zugleich beweisen, dass die Kultursparkassen vertrauenswürdig seien, weil sie kluge Investitionen tätigen und Werte schöpfen.

\section{Schlussfolgerungen}

In Nissens Revue wurde das Verhältnis der Varietäten zueinander sowohl konkret als auch in allegorischer Weise auf die Bühne gebracht. In personifizierter Form ,sprechen' nunmehr die Varietäten in wechselseitiger Ausrichtung aufeinander dies eine inkorporierte Darbietung der Machtverhältnisse und eine metapragmatische Kommentierung. Das Alltagsnähe suggerierende Gedicht von JohannsenBojsen besteht aus einem einzigen metapragmatischen Kommentar, wobei die 
Mehrsprachigkeitsrelationen von einer „wir“-Instanz thematisiert sowie zitathaft am Beispiel veranschaulicht werden. Die individuelle Aufwertung durch die Sprachenwahl, hier eine vorgestellte Standardvarietät des neu gewählten ,Mutterlandes', steht in unmittelbarer Abhängigkeit zur sozialen Mobilität.

Die beiden Beispiele können literarische Potentiale vergegenwärtigen: Durch Selbstreferenzialität und Hervorhebung des Konstruktcharakters werden die eingeschriebene Intentionalität sowie die Prozessualität und Pfadabhängigkeit der Registrierung in sprachlichen Äußerungen nachvollzogen. Erzählinstanzen oder Figuren führen hier mit einer gewissen Notwendigkeit vor, dass Begriffe und Konzeptualisierungen sozialen Sprachgebrauchs niemals nicht-ideologisch verwendbar sind. Im Gegenzug scheinen literarische Texte nur unter spezifischen Bedingungen für eine derart intensive Verhandlung von Varietäten-Konstellationen offen, denn über ideologische Topoi oder Stereotypisierungen sind selbst Autorinnen und Autoren mit Expertise und einschlägigen Lebenserfahrungen keineswegs erhaben.

Die thematisierte Mehrsprachigkeit und die sprachliche Verfasstheit von Nissens Revue verhalten sich dabei paradox zueinander, denn die dänische Standardvarietät wird in ihrem ranghöchsten Status bestätigt. Vor diesem Hintergrund scheinen altbekannte nationalsprachliche Konkurrenzmuster wiederbelebt. Diese Bestärkung des Standard-Dänischen als Literatursprache korrespondiert fast möchte man dies bedauern - mit der Funktionalisierung der „Südschleswiger“ Literatur als Erinnerungsliteratur, wie sie u. a. von Johannsen-Bojsen lanciert worden ist. Mit dieser Neueinbettung könnte sich eine Schließungsbewegung durch ,Monumentalisierung ' (statt einer fortgesetzt dynamisierenden und mehrsprachlichen Aushandlung) abzeichnen.

Auf der Performanzebene wird in beiden ausgewählten Texten code-switching als stilistisches Verfahren demonstriert. Eine weitere, bisher kaum beachtete Gemeinsamkeit ist der Nachvollzug von Lernersprache im Rahmen der vorgeführten Spracharbeit. Im Hinblick auf die eingangs genannten Grundfunktionen der Kontinuitätsverlängerung und des Institutionenerhalts wird damit ein sprachideologisch brisantes Verhandlungsfeld eröffnet. Durch die Revitalisierung von Sønderjysk, wie sie in der Revue problematisiert erscheint, ist wiederum möglicherweise einerseits eine Wiedererstarkung der Lokalliteratur in einem reterritorialisierten Sinne angezeigt, andererseits wird der transnationale Sprachgebrauch nachdrücklich hervorgehoben. Das monolinguale Paradigma hat in einem dezidiert mehrsprachlichen Verhandlungsfeld jedenfalls keine Zukunft. 


\section{Literaturverzeichnis}

Agha, Asif. Language and Social Relations. Cambridge: Cambridge University Press, 2007. Andersen, Signe, und Maike Lohse (Hg.). Sydslesvig Antologi. Tønder: Forlaget Neffen, 2015. Davidsen, Hans Christian. „Kai Nissen“. http://www.litteratursiden.dk/blogs/hans-christiandavidsen/20140908-0 (Weblog, 23.04.2016) [Material nicht mehr zugänglich].

„Erklärung der Regierung der Bundesrepublik Deutschland“. 1955. http://www.kopenhagen. diplo.de/contentblob/3823182/Daten/3054112/bonnererklaerung.pdf (Weblog, 2. November 2017).

Fredsted, Elin. „Language contact in the German-Danish border area in the twenty-first century“. STUF - Language Typology and Universals 69.3 (2016): 437-465. https://www. degruyter.com/view/j/stuf.2016.69.issue-3/stuf-2016-0018/stuf-2016-0018.xml (Weblog, 2. November 2017).

Johannsen, Karin. „Sproget“. Sindelag. Digte. Flensburg: Dansk Centralbibliotek for Sydslesvig, 1977. 80-81.

Johannsen-Bojsen, Karin. „Sagen fremfor alt - eller også personen?“ Kontakt, 3.3.2016. http:// syfo.de/fileadmin/syfo/KONTAKT/KONTAKT_3.3.2016.pdf (Weblog, 2. November 2017).

Nissen, Kaj. Søsteren der ikke vendte hjem. Et stykke om Sydslesvig. Flensburg: Det lille Teater, 2016. http://www.detlilleteater.de/sosteren.pdf (Weblog, 24. März 2016) [Manuskript nicht mehr zugänglich].

Spitzmüller, Jürgen. „Sprachideologie und/als soziale Positionierung“. Vortrag an der Uni Wien, 3. Juni 2014. Handout. http://www.spitzmueller.org/docs/handout-wien-2014-06-03.pdf (Weblog, 2. November 2017), sowie PowerPoint-Präsentation Sprachideologie und/als soziale Positionierung. http://www.spitzmueller.org/docs/praes-wien-2014-06-03.pdf (Weblog, 2. November 2017).

Sternberg, Meir. „Polylingualism as reality and translation as mimesis“. Poetics Today. Translation Theory and Intercultural Relations 2.4 (1981): 221-239. http://www.jstor.org/ stable/1772500 (Weblog, 2. November 2017).

Antje Wischmann ist Professorin an der Abteilung Skandinavistik, Universität Wien, nach Tätigkeiten als Dozentin und Forscherin in Huddinge bei Stockholm sowie als Gast- und Vertretungsprofessorin am Nordeuropa-Institut, HU Berlin, und an der Eberhard Karls Universität Tübingen. Forschungsgebiete: Skandinavische Literatur des 19. bis 21. Jahrhunderts u. a. mit den Schwerpunkten Gegenwartsliteratur, kulturwissenschaftlich erweiterte Zugänge zur Skandinavistik, Urbanistik, Mobilität. 